\title{
Review of: "The impact of hyperglycemia upon BeWo trophoblast cell metabolic function: A multi-OMICS and functional metabolic analysis"
}

\author{
Katsuhiko Takahashi ${ }^{1}$ \\ 1 Hoshi University
}

Potential competing interests: The author(s) declared that no potential competing interests exist.

Easton et al's manuscript shows the data of comprehensive analysis of BeWo cells under hyperglycemic (4500mg glucose/L) condition.

As authors introduced, they experimentally set hyperglycemia to mimic gestational diabetes (GDM). After all, the authors examined the effects of hyperglycemia on undifferentiated (CT) BeWo cell. So I could interpret the multi-OMICS analysis data could be useful for pre-existing DM among GDM.

Figure $1 \sim 7$

There are multi comparison, that is, low glucose versus high glucose condition, and undifferentiation (CT) and differentiation (SCT). Overall, the results and figures had many negative contents in hyperglycemia's action. The latter of the results treated the OMICS analysis in CT BeWo culture. The SCT's data would be excluded or be moved as supplement figs.

In Fig2, the authors showed "cell viability", but was there died cells in the conditions significantly? I want the authors to present the growth curve (for example, $X$; culture days, $Y$; cell number) to indicate the difference of growth rate in each culture condition.

In Fig3, the 3 contents are the SCT differentiation markers. Fig3D is interesting, but the manuscript displayed no OMICS analysis of SCT BeWo.

In Fig4, Fig4D is only positive graph. And Table 2 and 3 showed that hyperglycemia did not significantly affect on mitochondrial respiratory activity. Does the expression level alteration of COX II, a component of ETC complex IV, have any important means?

Though Fig5A has "2WA: Differentiation state", parhaps Fig5B should be written. Please check the graphs. Fig6 and 7 are main data of the authors. In "results", the authors should mention the reasons to detect the phosphorylated molecules (Glycogen synthase and GSK3be-ta). Fig7B has no differentiation state.

Table1 and 2 are not coincident to "result". I think Table1 and 2 may be reverse.

Fig. 8-13

In general, it's a too rough way of showing results to understand the contents. I guess that the authors just pasted in the results of the outsourced analysis. 
What was revealed by the OMICS analysis? Fig10 is the summary for Fig 8 and 9 . The letters of both Fig 10 A,B are too small to read at all. Is Fig 10A negative? And available information is the top (Overview of Nanoparticle Effects Pathway) of Fig 10B, isn't it? Actually, Page19 line408-410 has the explanation of alteration of the related genes levels. Which figures will the readers see? And what was indicated from Fig $11-13 ?$

The authors should indicate the important metabolites in the hyperglycemic cultured BeWo cells. Please link the results out of Fig 2-7, Fig 8-10, and Fig 11-13 to discuss the prevention and treatment against GDM. 